\title{
On the Steady-State Performance of Kalman Filtering with Intermittent Observations for Stable Systems
}

\author{
Ali Vakili and Babak Hassibi
}

\begin{abstract}
Many recent problems in distributed estimation and control reduce to estimating the state of a dynamical system using sensor measurments that are transmitted across a lossy network. A framework for analyzing such systems was proposed in [1] and called Kalman filtering with intermittent observations. The performance of such a system, i.e., the error covariance matrix, is governed by the solution of a matrixvalued random Riccati recursion. Unfortunately, to date, the tools for analyzing such recursions are woefully lacking, ostensibly because the recursions are both nonlinear and random, and hence intractable if one wants to analyze them exactly. In this paper, we extend some of the large random matrix techniques first introduced in [2], [3] to Kalman filtering with intermittent observations. For systems with a stable system matrix and i.i.d. time-varying measurement matrices, we obtain explicit equations that allow one to compute the asymptotic eigendistribution of the error covariance matrix. Simulations show excellent agreement between the theoretical and empirical results for systems with as low as $n=10,20$ states. Extending the results to unstable system matrices and time-invariant measurement matrices is currently under investigation.
\end{abstract}

\section{INTRODUCTION}

Groundbreaking advances in microsensor technology in the past decade have made several previously out of reach applications feasible. The proposed and/or already deployed applications include but are not limited to distributed catastrophe surveillance, smart transportation systems, and more efficient electric power grids. All these applications essentially rely on large scale networks that incorporate communication, estimation, and control. Therefore a significant body of research in recent years has been devoted to the study of distributed estimation and control over networks of many sensors and actuators. An important aspect of these systems which has prohibited direct extension of the classical theories of control and estimation is the natural unreliability of the underlying communication links. The stingy power constraints of microsensors only worsen the situation by making reliable communication further unrealistic.

A very well-received model for studying the effect of unreliable links in distributed sensing and control problems ([1], [4], [5], [6], [7]) assumes that the estimation and control data are in the form of packets which travel through an erasure network and each packet may be independently lost

This work was supported in part by the National Science Foundation under grant CCF-0729203, by the David and Lucille Packard Foundation, and by Caltech's Lee Center for Advanced Networking.

Ali Vakili is with Electrical Engineering Department, California Institute of Technology, 1200 E California Blvd., Pasadena, CA 91125, USA avakili@caltech.edu

Babak Hassibi is with Faculty of Electrical Engineering Department, California Institute of Technology, 1200 E California Blvd., Pasadena, CA 91125, USA hassibiecaltech.edu according to some probability. This model makes many problems mathematically tractable and at the same time has great resemblance with practice. In the very heart of this approach lies Kalman filtering with intermittent observations.

More formally, we have a linear, but time-varying, statespace model of the form,

$$
\begin{aligned}
& \left\{\begin{aligned}
x_{i+1} & =\mathrm{F} x_{i}+u_{i} \\
y_{i} & =\mathrm{H} x_{i}+v_{i}
\end{aligned}\right. \\
& \mathbb{E}\left[\begin{array}{c}
u_{i} \\
v_{i}
\end{array}\right]\left[\begin{array}{ll}
u_{j}^{*} & v_{j}^{*}
\end{array}\right]=\left[\begin{array}{cc}
\mathrm{Q} & 0 \\
0 & \mathrm{R}_{i}
\end{array}\right] \delta_{i j}
\end{aligned}
$$

where $x_{i}$ is the $n$-dimensional state vector, $y_{i}$ is the $m$ dimensional measurement vector, and $u_{i}$ and $v_{i}$ are zeromean process and measurement noises. All the measurements are assumed to have the same noise variance and to be independent of each other. Each component of the measurement vector may also be lost independently over both measurements and time with some fixed packet drop probability, $p_{d}$. Therefore the measurement noise covariance matrix should be represented by a matrix-valued i.i.d. random matrix process. In fact, the only thing time-varying about (12 ) is the noise covariance $\mathbf{R}_{i}$.

The estimation error covariance of the Kalman filter for the above system can be shown to satisfy a random Riccati recursion,

$$
\mathrm{P}_{i+1}=\mathrm{FP}_{i} \mathrm{~F}^{*}+\mathrm{Q}-\mathrm{FP}_{i} \mathrm{H}^{*}\left(\mathrm{R}_{i}+\mathrm{HP}_{i} \mathrm{H}^{*}\right)^{-1} \mathrm{HP}_{i} \mathrm{~F}^{*} .
$$

Clearly, in contrast to the classic case of time-invariant Kalman filtering, the above Riccati recursion does not converge to any specific value. The reason being that the covariance matrix $\mathbf{R}_{i}$ is indeed random and time-varying. However, there are several important questions that may be asked about such a recursion, especially about the distribution of the eigenvalues of $\mathrm{P}_{i}$.

Since $\mathrm{R}_{i}$ is a matrix-valued stationary random process, it may be expected that $\mathrm{P}_{i}$ also converges to a stationary process. Furthermore, one can argue that the state vector size is usually large due to the fact that the dynamical systems under consideration are often complex. This allows the use of several powerful tools which have been developed in the theory of large random matrices. In this work, we find the eigenvalue distribution of the prediction error covariance under these two assumptions. We will make two further assumptions. The first is that the measurement matrix $\mathrm{H}$ is also time-varying and random. In this sense, we will be forced to depart from the model (1-2). The main reason is that we do not quite yet know how to extend our techniques to deal with a fixed H. Nonetheless, as will be explained later 
in the paper, this assumption, and the relation to the constant $\mathrm{H}$ case, can be justified through the ergodicity of random matrix ensembles. The second assumption is that the matrix $\mathrm{F}$ is stable. This implies that the matrix-valued process $\mathrm{P}_{i}$ is bounded and thus we do not need to worry about stability in our analysis. How to relax these two assumptions is currently under investigation.

The problem of Kalman filtering with intermittent observations was first considered in [1] where the authors find upper and lower bounds on the critical packet drop probability above which the Kalman filter diverges. The tightness of the lower bound is further investigated in [8]. In [9] the authors characterize the critical packet drop probability for boundedness of the error covariance for a wide range of systems. Other authors have studied various sensor data transmission scenarios [10], [11], [12].

The rest of the paper is organized as follows. In Section II we will give a brief overview of definitions and tools from random matrix theory which are used in this work. Section III contains the main results of the paper. We look at two scenarios for the process noise covariance matrix, namely, when $\mathrm{Q}$ is a multiple of identity or a Wishart matrix. We find the steady-state eigendistribution of $\mathrm{P}_{i}$ as the solution of a pair of implicit equations. Simulation results provided show the accuracy of our method in predicting the eigendistribution. Finally, Section IV concludes the paper.

\section{Pertinent Results From Random Matrix THEORY}

In this section we give a brief overview of pertinent definitions and results from random matrix theory. A more comprehensive review of the subject can be found in [13], [14]. A random matrix is simply described by the joint probability distribution of its entries. Often, the most important questions to be answered about a random matrix ensemble concern the distribution of the eigenvalues. For an $n \times n$ random matrix, $M$, the empirical cumulative distribution function of the eigenvalues is defined as,

$$
F_{\mathrm{M}}(\lambda)=\frac{1}{n} \sum_{l=1}^{n} \operatorname{Pr}\left\{\lambda_{l}(\mathrm{M}) \leq \lambda\right\},
$$

where $\lambda_{l}(\mathbf{M})$ denotes the $l$-th eigenvalue of $\mathbf{M}$. An empirical density function, $f_{M}(x)$, can be associated with $F_{\mathrm{M}}(x)$. This density function is frequently referred to as the eigendistribution of $\mathrm{M}$ and is nothing but the marginal distribution of one randomly selected eigenvalue of one realization of the random matrix. As will be explained in the next section, finding the eigendistribution of the error covariance matrices under consideration is of significant importance. It turns out that in the random matrix arena, results on the eigendistribution of random matrices can be expressed much more efficiently in terms of certain transforms of $f_{M}(\lambda)$. The most ubiquitous example of these transforms is the so-called Stieltjes transform which was first used in the seminal work of Marcenko and Pastur [15]. The Stieltjes transform of the eigendistribution of a random matrix $\mathbf{M}$-interchangeably referred to as the Stieltjes transform of the matrix itselfis defined on the complex plane as,

$$
S_{\mathrm{M}}(z)=\mathbb{E}\left[\frac{1}{\lambda-z}\right]=\int \frac{f_{\mathrm{M}}(\lambda)}{\lambda-z} d \lambda .
$$

Having the Stieltjes transform, the eigendistribution can be uniquely retrieved through its inversion formula [16],

$$
f_{\mathrm{M}}(\lambda)=\lim _{\omega \rightarrow 0^{+}} \frac{1}{\pi} \operatorname{Im}\left[S_{\mathrm{M}}(\lambda+j \omega)\right] .
$$

The main reason for the Stieltjes to be a handy tool in random matrix theory is that it can be directly computed from the random matrix itself through an alternative definition,

$$
S_{\mathrm{M}}(z)=\mathbb{E} \frac{1}{n} \operatorname{tr}(\mathrm{M}-z \mathrm{I})^{-1},
$$

or equivalently,

$$
S_{\mathrm{M}}(z)=-\frac{d}{d z} \mathbb{E} \frac{1}{n} \log \operatorname{det}(\mathrm{M}-z \mathrm{I}) .
$$

As can be clearly seen through the above definition, the Stieltjes transform can be computed without finding the eigenvalues themselves. Then, having $S_{\mathrm{M}}(z)$, the eigendistribution can be easily found through (6).

Another important aspect of random matrix theory is the set of powerful tools available for analyzing large random matrices. While the majority of results on the eigendistribution of fixed-dimensional random matrices are complicated and offer little insight -and mainly limited to Gaussian random matrices and matrices derived from them-, when the matrix dimensions are allowed to grow, one usually finds simple, closed form expressions for the eigendistribution that behave like universal laws, i.e., they depend on the matrix structure and statistics rather than the exact distribution of the entries. Moreover, although the derivations are carried out for $n \gg 1$, the law of large numbers guarantees fast convergence to the asymptotic results for $\mathrm{n}$ being as small as $10-20$. This range of values for the state vector size is reasonable in most applications. Moreover, random matrix ensembles demonstrate an ergodic behavior in the sense that every single realization looks similar to the deterministic asymptotic eigendistribution. In terms of eigenvalues, it means that $F_{\mathrm{M}}(\lambda)$ in (4) does not change if we replace the $\operatorname{Pr}(\cdot)$ with the Heaviside step function, $\mathbb{1}(\cdot)$.

A useful property of the Stieltjes transform in the asymptotic regime is the so-called self-averaging property which is stated in the follwing Lemma [17]:

Lemma 2.1 (Self-Averaging): Let $\mathrm{M}$ be an $n \times n$ positive semidefinite random matrix. If the empirical eigendistribution of $\mathrm{M}$ almost surely converges to its mean value as $n \rightarrow \infty$, i.e.,

$$
\lim _{n \rightarrow \infty} \frac{1}{n} \operatorname{tr}(\mathrm{M}-z \mathrm{I})^{-1}=S_{\mathrm{M}}(z) \quad \text { a.s. }
$$

(Note the absence of the expectation) then for any $n$ dimensional vector $x$ independent of $M$ with i.i.d. zero-mean, unit-variance elements with bounded higher moments, we have,

$$
\lim _{n \rightarrow \infty} \frac{1}{n} x^{T}(\mathrm{M}-z \mathrm{I})^{-1} x=S_{\mathrm{M}}(z) \quad \text { a.s. }
$$


The above Lemma can be intuitively verified by comparing to the definition (7).

\section{Kalman Filtering With Intermittent OBSERVATIONS}

In this section we consider Kalman filtering with intermittent measurements under the assumption of a stable system matrix and a time-varying observation matrix. Although this is not the convention in the literature, there are several reasons for doing so. The first is that we do not yet know how to deal with the time-invariant case in our method. The second reason is that in many recent applications of distributed estimation, the sensors' environment and/or their position is time-varying. Finally, as mentioned earlier, in the theory of large random matrices every single realization of an ensemble has an eigendistribution that converges almost surely to the asymptotic eigendistribution. Therefore one may expect that if the matrix $\mathrm{H}$ is large enough, then the eigendistribution of $\mathrm{P}_{i}$ may be the same irrespective of $\mathrm{H}$ and whether it is time-varying. The second assumption is that the matrix $\mathrm{F}$ is stable. As mentioned in the introduction, this guarantees the boundedness of $\mathrm{P}_{i}$ and will absolve us of having to consider stability issues. Of course, relaxing these two assumptions is critical and currently under investiagtion. In this sense, the results reported in this paper represent significant progress towards analyzing these more general and realistic cases.

In the following, we will consider two cases in which the state process noise covariance matrix is either a multiple of identity or a Wishart matrix, where the latter means $\mathrm{Q}=$ $\mathrm{GG}^{*}$ where $\mathrm{G}$ is an $n \times m_{G}$ matrix with i.i.d. zero-mean, $\frac{1}{\sqrt{m_{G}}}$-variance entries.

For each Riccati recursion, we will find the steady-state eigendistribution of $\mathrm{P}_{i}$. There are several reasons to look at the eigendistribution. First of all, $\mathbb{E}[\lambda]=\frac{1}{n} \mathbb{E}\left[\operatorname{tr} P_{i}\right]$ is nothing but the mean square error performance of Kalman filtering. Moreover, determining the support of eigendistribution is crucial for finding various performance bounds and studying the system stability. On the other hand, the convergence properties of the eigendistribution in the transient phase directly establish the convergence properties of the recursion itself. Thus in this work we focus on characterizing the eigendistributions in the steady state. The transient behavior is well worth of future scrutiny.

\section{A. The case of $Q=q I$}

When $\mathrm{Q}=q \mathrm{I}$ in the linear time-varying state-space model under study, the error covariance matrix undergoes a random Riccati recursion of the form,

$$
\mathrm{P}_{i+1}=\alpha \mathrm{F}\left(\mathrm{P}_{i}^{-1}+\mathrm{H}_{i}^{*} \mathrm{R}_{i}^{-1} \mathrm{H}_{i}\right)^{-1} \mathrm{~F}^{*}+q \mathrm{I}, \quad \mathrm{P}_{0},
$$

in which $\alpha$ is a scalar between 0 and 1 , and $\mathrm{F}$ is assumed to be an $n \times n$ matrix with i.i.d. entries having zero mean and variance $\frac{1}{\sqrt{n}}$. The observation matrix, $\mathrm{H}_{i}$, is an $m \times n$ matrix with i.i.d. zero-mean $\frac{1}{\sqrt{m}}$-variance entries and $q$ is a constant denoting the variance of the state process noise. As mentioned in Section. I, we will assume that the observations may independently be missing with some probability $p_{d}$. Thus $\mathrm{R}_{i}$ can be modeled as a diagonal matrix with independent entries such that,

$$
\left(\mathrm{R}_{i}^{-1}\right)_{j j}=\left\{\begin{array}{ll}
0 & \text { with probability } p_{d} \\
\frac{1}{r} & \text { with probability } 1-p_{d}
\end{array} .\right.
$$

We are interested in finding the steady-state, i.e. when $i \rightarrow \infty$, eigendistribution of the error covariance matrix $\mathrm{P}_{i}$, or equivalently its Stieltjes transform. It can be shown that the Stieltjes transform at the steady-state satisfies a set of implicit equations on the complex plane:

Theorem 3.1: Let $\mathrm{F}$ be an $n \times n$ matrix with i.i.d. entries having zero mean and variance $\frac{1}{\sqrt{n}}$ and $\alpha$ to be such that $\sqrt{\alpha} \mathrm{F}$ is stable. As $i \rightarrow \infty$ and $n \rightarrow \infty$, the eigendistribution of $\mathrm{P}_{i}$ in (11) converges to a stationary distribution whose Stieltjes transform, $S_{\mathrm{P}}(z)$, satisfies (12) and (13),

$$
\begin{aligned}
S_{\mathrm{P}}(z) & =-\frac{1}{z-q}+\frac{\alpha r^{\prime} S_{\mathrm{P}}(z)}{z-q} \Omega\left(-\alpha r^{\prime} S_{\mathrm{P}}(z)\right), \\
\Omega(z) & =-\frac{1}{r^{\prime} u(z)}-\frac{1}{r^{\prime} u^{2}(z)} S_{\mathrm{P}}\left(\frac{1}{u(z)}\right),
\end{aligned}
$$

in which $u(z)$ is an expression in terms of $z$ and $\Omega(z)$,

$$
u(z)=\frac{z}{r^{\prime}}-\frac{\beta^{\prime} / r^{\prime}}{\beta^{\prime}+\Omega(z)},
$$

which is used here for the sake of brevity in the expressions, $\beta^{\prime}=\frac{\left(1-p_{d}\right) m}{n}$, and $r^{\prime}=\frac{r}{1-p_{d}}$.

Proof: We mention that the proof presented is at a somewhat high level; although it can be made rigorous, we shall not do so for reasons of space.

First of all, note that with high probability the term $\mathrm{H}_{i}^{*} \mathrm{R}_{i}^{-1} \mathrm{H}_{i}$ can be written as $\frac{\left(1-p_{d}\right)}{r} \overline{\mathrm{H}}_{i}^{*} \overline{\mathrm{H}}_{i}$ where $\overline{\mathrm{H}}_{i}$ is an $m\left(1-p_{d}\right) \times n$ matrix with i.i.d. entries having zero mean and $\frac{1}{\sqrt{m\left(1-p_{d}\right)}}$ variance. Now we can rewrite (11) as,

$$
\mathbf{P}_{i+1}=\alpha r^{\prime} \mathrm{F}\left(r^{\prime} \mathbf{P}_{i}^{-1}+\overline{\mathbf{H}}_{i}^{*} \overline{\mathrm{H}}_{i}\right)^{-1} \mathrm{~F}^{*}+q \mathrm{I},
$$

where,

$$
r^{\prime}=\frac{r}{1-p_{d}}
$$

Using the definition of the Stieltjes transform (7),

$$
S_{\mathrm{P}, i+1}(z)=\frac{1}{\alpha r^{\prime}} S_{\mathrm{B}, i}\left(\frac{z-q}{\alpha r^{\prime}}\right),
$$

in which we have used $\mathrm{B}_{i}$ to denote,

$$
\mathrm{B}_{i}=\mathrm{F}\left(r^{\prime} \mathrm{P}_{i}^{-1}+\overline{\mathrm{H}}_{i}^{*} \overline{\mathrm{H}}_{i}\right)^{-1} \mathrm{~F}^{*} .
$$

Therefore we focus on finding $S_{\mathrm{B}, i}(z)$. Instead of the formal and lengthy proof, hereby we give a sketch of the argument. A fundamental concept in random matrix theory is the notion of free probability which was first introduced by Voiculescu [18], [19]. Freeness replaces the independence notion for random variables that are non-commutative, as are the random matrices. Essentially two non-commutative random variables $X$ and $Y$ are called free if,

$$
\mathbb{E}\left[p_{1}(X) q_{1}(Y) p_{2}(X) q_{2}(Y) \ldots\right]=0,
$$


for finitely many polynomials $p_{i}$ and $q_{j}$ for which,

$$
\mathbb{E}\left[p_{i}(X)\right]=0=\mathbb{E}\left[q_{j}(Y)\right] .
$$

For free random variables, there exists a useful transform called S-transform which is defined based on the Stieltjes transform as,

$$
\Sigma(\gamma)=-\frac{1}{\gamma} S\left(\frac{\gamma+1}{\gamma \Sigma(\gamma)}\right)
$$

S-transform is the analog of the so-called Mellin transform for pairs of commutative random variables, in the sense that for two free random variables $X$ and $Y$,

$$
\Sigma_{\mathrm{AB}}(\gamma)=\Sigma_{\mathrm{A}}(\gamma) \Sigma_{\mathrm{B}}(\gamma) .
$$

Therefore if one can establish the freeness of two noncommutative random variables, the Stieltjes transform of their product can be expressed in terms of the individual Stieltjes transforms in an implicit form. Now going back to (18), according to the definition (7), it makes no difference to look at the Stieltjes transform of $\mathrm{F}^{*} \mathrm{~F}\left(r^{\prime} \mathrm{P}_{i}^{-1}+\overline{\mathrm{H}}_{i}^{*} \overline{\mathrm{H}}_{i}\right)^{-1}$. Moreover, we can diagonalize $\mathrm{P}_{i}$ without loss of generality since the distributions of $\mathrm{H}_{i}$ and $\mathrm{F}$ are isotropic. Now the results of [20] can be used to establish the freeness of $\mathrm{F}^{*} \mathrm{~F}$ and $\left(r^{\prime} \mathrm{P}_{i}^{-1}+\overline{\mathrm{H}}_{i}^{*} \overline{\mathrm{H}}_{i}\right)^{-1}$ in the steady-state. To do so it is necessary to use random dynamical systems theory [21] to establish the existence of a unique steady-state eigendistribution for $\mathrm{P}_{i}$ [22]. Then it is straightforward to show that for this choice of $\mathrm{F}$,

$$
\Sigma_{\mathrm{F} * \mathrm{~F}}(\gamma)=\frac{1}{1+\gamma} .
$$

Therefore, using (22), (23), and the definition of the Stransform, we can show that,

$$
S_{\mathrm{B}}(z)=-\frac{1}{z}-\frac{S_{\mathrm{B}}(z)}{z} \Omega\left(-S_{\mathrm{B}}(z)\right),
$$

(please note the absence of the index $i$ due to the steadystate analysis from now on,) where $\Omega_{i}(z)$ is just the Stieltjes transform of,

$$
\mathrm{A}_{i}=r^{\prime} \mathrm{P}_{i}^{-1}+\overline{\mathrm{H}}_{i}^{*} \overline{\mathrm{H}}_{i} .
$$

It only remains to find the relation between $\Omega_{i}(z)$ and the Stieltjes transform of $\mathrm{P}_{i}$ from the above equation. By applying the definition of the Stieltjes transform (7) to both sides we obtain,

$$
\Omega(z)=\frac{1}{n} \mathbb{E} \operatorname{tr}\left(r^{\prime} \mathrm{P}^{-1}+\overline{\mathrm{H}}^{*} \overline{\mathrm{H}}-z \mathrm{I}\right)^{-1} .
$$

Since $\overline{\mathrm{H}}$ has an isotropic distribution, without loss of generality, $\mathrm{P}^{-1}$ can be assumed to be a diagonal matrix, $\Lambda$. Now we break $\Lambda$ and $\overline{\mathrm{H}}$ in the form,

$$
\Lambda=\left[\begin{array}{cc}
\lambda_{1} & 0 \\
0 & \Lambda_{2}
\end{array}\right] \quad, \quad \overline{\mathrm{H}}=\left[\begin{array}{ll}
h_{1} & \mathrm{H}_{2}
\end{array}\right],
$$

and rewrite the RHS of (26) as,

$$
\frac{1}{n} \mathbb{E} \operatorname{tr}\left[\begin{array}{cc}
r^{\prime} \lambda_{1}+h_{1}^{*} h_{1}-z & h_{1}^{*} \mathrm{H}_{2} \\
\mathrm{H}_{2}^{*} h_{1} & r^{\prime} \Lambda_{2}+\mathrm{H}_{2}^{*} \mathrm{H}_{2}-z \mathrm{I}
\end{array}\right]^{-1}
$$

Clearly, since we are interested in the marginal eigendistributions, it does not matter which diagonal entry of the above inverse we look at. Thus, without loss of generality, we can focus on the first diagonal entry which can be written as,

$$
\mathbb{E} \frac{1}{r^{\prime} \lambda_{1}-z+h_{1}^{*}\left(\mathrm{I}-\mathrm{H}_{2}\left(r^{\prime} \Lambda_{2}-z \mathbf{I}+\mathrm{H}_{2}^{*} \mathrm{H}_{2}\right)^{-1} \mathrm{H}_{2}^{*}\right) h_{1}} .
$$

Using the matrix inversion lemma, we can rewrite the above expression as,

$$
\mathbb{E} \frac{1}{r^{\prime} \lambda_{1}-z+h_{1}^{*}\left(\mathrm{I}+\mathrm{H}_{2}\left(r^{\prime} \Lambda_{2}-z \mathrm{I}\right)^{-1} \mathrm{H}_{2}^{*}\right)^{-1} h_{1}} .
$$

Now one can invoke the self-averaging Lemma (9) -using the independence of $h_{1}$ and the inversed matrix in the denominator- to find that,

$$
\Omega(z)=\mathbb{E} \frac{1}{r^{\prime} \lambda_{1}-z+\underbrace{\frac{1}{m^{\prime}} \mathbb{E} \operatorname{tr}\left(\mathrm{I}+\mathrm{H}_{2}\left(r^{\prime} \Lambda_{2}-z \mathrm{I}\right)^{-1} \mathrm{H}_{2}^{*}\right)^{-1}}_{\triangleq w(z)}},
$$

where $m^{\prime}=m\left(1-p_{d}\right)$. In order to find $w(z)$, we employ the same technique used to obtain (31) by breaking $\mathrm{H}_{2}$ in the form,

$$
\mathrm{H}_{2}=\left[\begin{array}{c}
h_{21} \\
\mathrm{H}_{22}
\end{array}\right],
$$

which eventually yields,

$$
w(z)=\mathbb{E} \frac{1}{1+\underbrace{h_{21}\left(r \Lambda_{2}+\mathrm{H}_{22}^{*} \mathrm{H}_{22}-z \mathrm{I}\right)^{-1} h_{21}^{*}}_{\triangleq_{w^{\prime}}(z)}},
$$

where, by invoking the self-averaging lemma, $w^{\prime}(z)$ in turn can be rewritten as,

$$
w^{\prime}(z)=\frac{n}{m^{\prime}} \times \frac{1}{n} \operatorname{tr}\left(r \Lambda_{2}+\mathrm{H}_{22}^{*} \mathrm{H}_{22}-z \mathrm{I}\right)^{-1} .
$$

The second part of the expression above is nothing but an $(n-1) \times(n-1)$ version of (26). Since we are considering the large $n$ regime, $w^{\prime}(z)$ can be simplified as $\frac{n}{m^{\prime}} \Omega(z)$. Therefore,

$$
w(z)=\frac{1}{1+\frac{n}{m^{\prime}} \Omega(z)}
$$

We can now replace (34) into (31). Since $\lambda_{1}$ is a randomly selected eigenvalue of $\mathrm{P}^{-1}$, (31) can be written in terms of the Stieltjes transform of $\mathrm{P}^{-1}$,

$$
\Omega(z)=\frac{1}{r^{\prime}} S_{\mathrm{P}^{-1}}\left(\frac{z}{r^{\prime}}-\frac{\left(1-p_{d}\right) \beta / r^{\prime}}{\left(1-p_{d}\right) \beta+\Omega(z)}\right),
$$

which together with the relation between the Stieltjes transforms of a matrix and its inverse,

$$
S_{\mathrm{P}^{-1}}(z)=-\frac{1}{z}-\frac{1}{z^{2}} S_{\mathrm{P}}\left(\frac{1}{z}\right)
$$

result in (13). Substituting (24) in (17) yields (12) and completes the proof. 


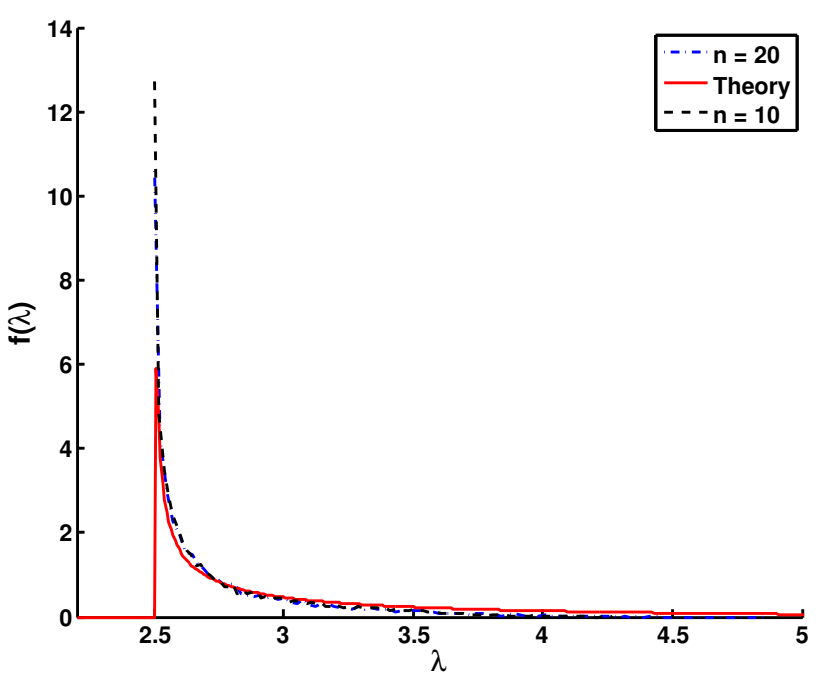

Fig. 1. The empirical steady-state eigendistribution of the recursion (11) for $n=10,20, m=30, r=0.5, q=2.5$, and $p_{d}=0.2$ compared to the theoretically found eigendistribution.

Since $\Omega(z)$ is a Stieltjes transform itself, equations (12) and (13) can be readily written in terms of the steady-state eigendistribution of $\mathrm{P}_{i}$ and an auxiliary probability distribution and be numerically solved to find the eigendistribution efficiently. In other words, the implicit equations of Theorem 3.1 need only be solved very close to the real line rather than on the whole complex plane. Figure 1 shows the simulation results for $n=10,20$ and $m=30$. The empirical curve is generated through Monte Carlo simulation of the recursion. It can be observed that the asymptotic theoretical prediction closely matches with the empirical curve for state vector size being as low as $n=10$.

It is also worth mentioning that the assumptions on the problem can be further relaxed by assuming that different measurements may have different packet drop probabilities, the average of whom equals $p_{d}$. This is often a more realistic model since in practice different sensors may be deployed at different locations and consequently have different channel strengths when they communicate through a wireless network.

\section{B. The case of $Q=G G^{*}$}

In order to extend the results to the case of state noise process covariance being a full matrix, here we consider $\mathrm{Q}$ being a Wishart matrix. As mentioned earlier, this means that,

$$
\mathrm{Q}=\mathrm{GG}^{*}
$$

where $\mathrm{G}$ is an $n \times m_{G}$ matrix with i.i.d. zero-mean, $\frac{1}{\sqrt{m_{G}}}-$ variance entries. The random Riccati recursion of the state estimation error covariance will be,

$$
\mathrm{P}_{i+1}=\alpha \mathrm{F}\left(\mathrm{P}_{i}^{-1}+\mathrm{H}_{i}^{*} \mathrm{R}_{i}^{-1} \mathrm{H}_{i}\right)^{-1} \mathrm{~F}^{*}+\mathrm{GG}^{*}, \quad \mathrm{P}_{0},
$$

where all the other parameters are as defined in the previous subsection. All the coefficients are time invariant except for the observation matrix $\mathrm{H}_{i}$ and observation noise covariance $\mathrm{R}_{i}$-of course, due to the intermittent observations,- and once again, we are interested in finding the steady-state eigendistribution of the error covariance matrix. The following theorem describes its Stieltjes transform as the solution of a pair of implicit equations:

Theorem 3.2: Let $\mathrm{F}$ and $\mathrm{G}$ be $n \times n$ and $n \times m_{G}$ matrices with i.i.d. entries having zero mean and variances $\frac{1}{\sqrt{n}}$ and $\frac{1}{\sqrt{m_{G}}}$, respectively, and $\alpha$ to be such that $\sqrt{\alpha} \mathrm{F}$ is stable. As $i \rightarrow \infty$, the eigendistribution of $\mathrm{P}_{i}$ in (11) converges to a stationary distribution whose Stieltjes transform, $S_{\mathrm{P}}(z)$, satisfies (39) and (40),

$$
\begin{aligned}
S_{\mathrm{P}}(z) & =-\frac{1}{\alpha r^{\prime} v(z)}+\frac{S_{\mathrm{P}}(z)}{v(z)} \Omega\left(-\alpha r^{\prime} S_{\mathrm{P}}(z)\right), \\
\Omega(z) & =-\frac{1}{r^{\prime} u(z)}-\frac{1}{r^{\prime} u^{2}(z)} S_{\mathrm{P}}\left(\frac{1}{u(z)}\right),
\end{aligned}
$$

in which $v(z)$ and $u(z)$ are expressions in terms of $z, S_{\mathrm{P}}(z)$, and $\Omega(z)$,

$$
\begin{aligned}
& v(z)=\frac{z}{\alpha r^{\prime}}-\frac{\beta_{G} / \alpha r^{\prime}}{\beta_{G}+S_{\mathrm{P}}(z)} \\
& u(z)=\frac{z}{r^{\prime}}-\frac{\beta^{\prime} / r^{\prime}}{\beta^{\prime}+\Omega(z)},
\end{aligned}
$$

which are used in order to simplify the equations, $\beta^{\prime}=$ $\frac{\left(1-p_{d}\right) m}{n}, r^{\prime}=\frac{r}{1-p_{d}}$, and $\beta_{G}=\frac{m_{G}}{n}$.

Sketch of proof: The proof essentially follows the proof of Theorem 3.1. The only difference is that instead of having,

$$
\mathrm{P}_{i}=\alpha r^{\prime} \mathrm{B}_{i}+q \mathrm{I},
$$

in this case we have,

$$
\mathrm{P}_{i}=\alpha r^{\prime} \mathrm{B}_{i}+\mathrm{GG}^{*},
$$

where $\mathrm{B}_{i}$ is as defined in (18). This is similar to the expression (25) that we dealt with in the proof of Theorem 3.1. Once again, although $\mathrm{G}$ is not time-varying, it is selected from an isotropic random matrix ensemble and therefore we can diagonalize $\mathrm{P}_{i}$ and follow the same techniques that where used to obtain (35) to show that,

$$
S_{\mathrm{P}, i+1}(z)=\frac{1}{\alpha r^{\prime}} S_{\mathrm{B}, i}\left(\frac{z}{\alpha r^{\prime}}-\frac{\beta_{G} / \alpha r^{\prime}}{\beta_{G}+S_{\mathrm{P}, i+1}(z)}\right) .
$$

Using (45) instead of (17), the rest of the proof will be similar to that of Theorem 3.1.

As in Theorem 3.1, this implicit pair of equations for $S_{\mathrm{P}}(z)$ can be rewritten as an expression for the steady-state eigendistribution involving an auxiliary distribution, which can be efficiently solved through numerical methods in order to determine the eigendistribution.

In Figure 2, we have plotted the theoretical curve obtained by numerically solving (39) and (40) versus the empirical eigendistributions which are found through Monte Carlo simulation of the recursion (38) for various values of $n$. It can be seen that the theoretical curve captures the behavior of the empirical one very closely. 


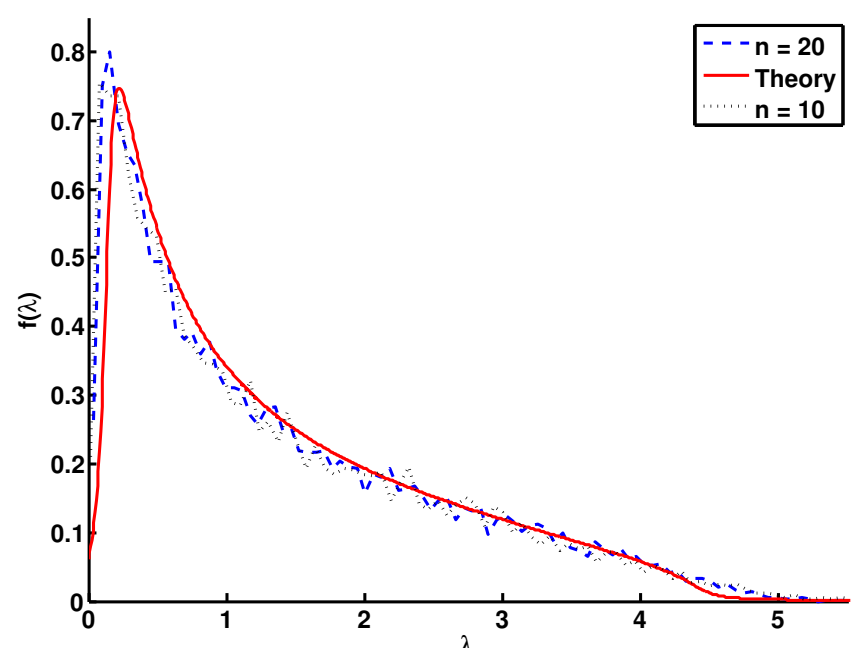

Fig. 2. The empirical steady-state eigendistribution of the recursion (38) for $n=10,20, m=30, r=0.5, q=2.5, m_{G}=n$, and $p_{d}=0.2$ compared to the theoretically found eigendistribution.

As mentioned in the previous subsection, our proof remains valid even when assuming that the observations are dropped independently with different probabilities, while the average of these packet drop probabilities equals $p_{d}$. In Figure 3, we have compared the theoretical curve with the Monte Carlo simulation results for this scenario. Each observation may be dropped with probability $p_{j}$, where $p_{j}$ 's are selected uniformly between zero and $2 p_{d}$.

\section{CONCLUSIONS}

Using the framework first introduced in [2], [3] we obtain explicit equations that allow one to compute the asymptotic eigendistribution of the error covariance matrix that arises in Kalman filtering with intermittent observations, when the state dimension is large. Our analysis relies on two key assumptions: the system matrix $\mathrm{F}$ is stable and the measurement matrices $\mathrm{H}_{i}$ are time-varying. Relaxing these assumptions, so that the results can apply to a wider range of problems, is currently under investigation.

\section{REFERENCES}

[1] B. Sinopoli, L. Schenato, M. Franceschetti, K. Poolla, M. Jordan, and S. Sastry, "Kalman filtering with intermittent observations," IEEE Transactions on Automatic Control, vol. 49, no. 9, pp. 1453-1464, Sep 2004.

[2] A. Vakili and B. Hassibi, "A Stieltjes transform approach for analyzing the RLS adaptive filter," in Proc. of 46th annual Allerton Conference on Communication, Control and Computing, September 2008.

[3] A. Vakili and B. Hassibi, "A stieltjes transform approach for studying the steady-state behavior of random lyapunov and riccati recursions," in Proc. of 47th IEEE Conference on Decision and Control, 2008.

[4] L. Schenato, B. Sinopoli, M. Franceschetti, K. Poolla, and S. Sastry, "Foundations of control and estimation over lossy networks," Proceedings of the IEEE, vol. 95, no. 1, pp. 163-187, Jan 2007.

[5] M. Epstein, L. Shi, A. Tiwari, and R. M. Murray, "Probabilistic performance of state estimation across a lossy network," in Automatica, 2008.

[6] O. C. Imer, S. Yuksel, and T. Basar, "Optimal control of dynamical systems over unreliable communication links," in Automatica, Sep. 2006, pp. 1429-1439.

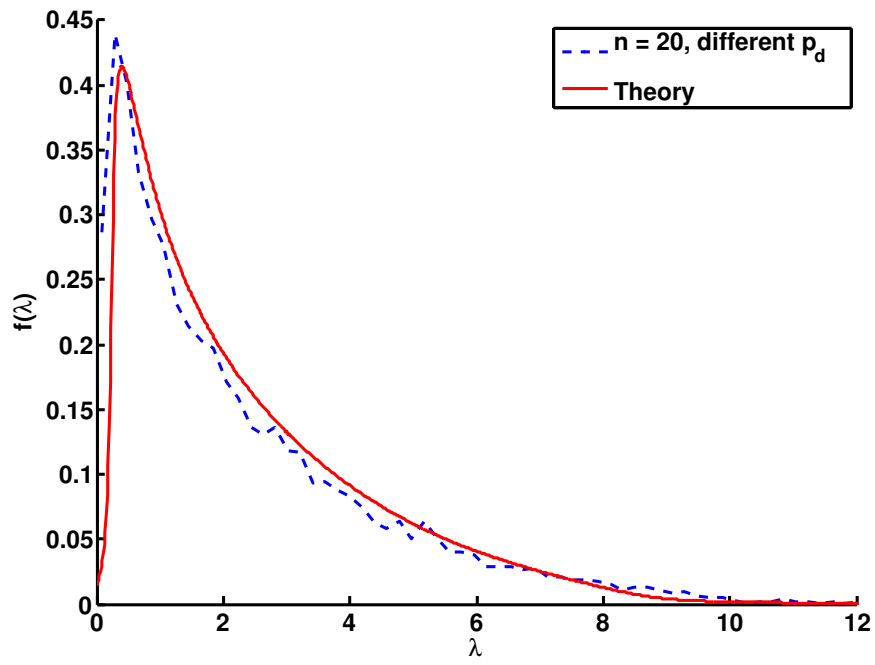

Fig. 3. The empirical steady-state eigendistribution of the recursion (38) for $n=10,20, m=30, r=1.5, q=2.5, m_{G}=n$, and $p_{d}$ uniformly distributed on $[0,0.4]$, compared to the theoretically found eigendistribution.

[7] V. Gupta, A. F. Dana, J. Hespanha, R. M. Murray, and B. Hassibi, "Data transmission over networks for estimation and control," IEEE Transactions on Automatic Control, submitted 2006.

[8] K. Plarre and F. Bullo, "On Kalman filtering for detectable systems with intermittent observations," IEEE Transactions on Automatic Control, May 2007, Submitted for publication.

[9] Y. Mo and B. Sinopoli, "A characterization of the critical value for kalman filtering with intermittent observations," in in proc. of 47 th IEEE Conference on Decision and Control.

[10] Y. Xu and J. Hespanha, "Estimation under controlled and uncontrolled communications in networked control systems," in Proc. of IEEE CDC-ECC, Dec. 2005, pp. 842-847.

[11] C. Robinson and P. R. Kumar, "Sending the most recent observation is not optimal in networked control: Linear temporal coding and towards the design of a control specific transport protocol," in Proc. of IEEE CDC, Dec. 2007, pp. 334-339.

[12] M. Huang and S. Dey, "Stability of Kalman filtering with Markovian packet drop," Automatica, vol. 43, no. 4, pp. 598-607, 2007.

[13] A. M. Tulino and S. Verdu, "Random matrix theory and wireless communications," Foundations and Trends in communications and Information Theory, vol. 1, no. 1, pp. 1-182, 2004.

[14] M. L. Mehta, Random Matrices, Prentice-Hall, 1991

[15] V. A. Marcenko and L. A. Pastur, "Distributions of eigenvalues for some sets of random matrices," Math. USSR-Sbornik, vol. 1, pp. 457483, 1967.

[16] J. Silverstein and S. Choi, "Analysis of the limiting spectral distribution of large dimensional random matrices," Journal of Multivariate Analysis, vol. 54, no. 2, pp. 295-309, 1995.

[17] Z. D. Bai and J. W. Silverstein, "No eigenvalues outside the support of the limiting spectral distribution of large dimensional sample covariance matrices," Annals of Probability, vol. 26, pp. 316-345, 1998.

[18] D. Voiculescu, "Asymptotically commuting finite rank unitary operators without commuting approximants," Acta Sci. Math., vol. 45, pp. 429-431, 1983.

[19] D. Voiculescu, "Addition of certain non-commuting random variables," Journal of Functional Analysis, vol. 66, 1986.

[20] O. Ryan, "On the limit distributions of random matrices with independent or free entries," Communications in Mathematical Physics, vol. 193, pp. 595-626, 1998.

[21] I. Chueshov, Monotone random systems - Theory and applications, Springer, 2002.

[22] B. Sinopoli et. al., "A random dynamical systems approach to networked control systems," in Information Theory and Applications Workshop, Feb. 2009 